Arch. hist. jap. Vol. 16, n. 2 (Fehruar 1959).

s. $237 \quad 249$.

Anat. Inst. d. Mediz. Fak., Tniv. Okayama (Vorstand: Prof. M. SEKI).

\title{
Vergleichende Untersuchungen über die Menge der Lipoide im Blutplasma und der Polysaccharidverbindungen im Knor- pel und über die Struktur der Aorta von Vögeln, Reptilien und Amphibien.
}

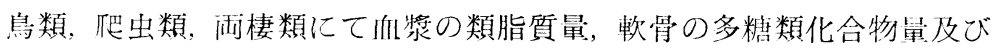
大動脉の構造の比較。

\author{
Toshikazu KITADE 北出俊一.
}

(Eingegangen am 30. November 1958.)

Nach der Untersuchung von KURITA (1955) wurde die durch langfristige Fiitterung mit einer an Cholesterin reichen Nahrung zu erzeugende Atherosklerose in der Aortenwand des Kaninchens durch eine gleichzeitige Injektion der Chondroitinschwefelsäure weitgehend verhindert, wobei sich der Cholesteringehalt im Blut auch weniger steigerte. Beim Huhn ersheint bekanntlich auch in normalen Zuständen oft eine Atherosklerose in der Aortenwand. Es wurde von DAUBER und KATZ (1942) gezeigt, daß beim Huhn bei Cholesterinzufuhr die Atherosklerose stärker vorkommt. MIYAO (1950) gab dem Huhn eine Cholesterin und Kottonöl enthaltene Nahrung und injizierte gleichzeitig Chondroitinschwefelsäure. Die Sklerosierung der Aorta und die Vermehrung von Cholesterin, Neutralfett und Lipoide im Blut wurden dann durch die Chondroitinschwefelsäure mehr oder weniger stark verhindert. Darauf, daß die alimentäre Lipämie sich durch die Injektion mit dem der Chondroitinschwefelsäure verwandten Heparin vermeiden läßt. hat shon HAHN (1943) beim Hund aufmerksam gemacht. Es bestehe also ein Antagonismus zwischen Mucopolysaccharid und Lipoiden im Tierkörper. Die vorliegende Mitteilung befaßt sich mit der im Teil zum Ausdruck gebrachten Aufgaben an verschiedenen Tierarten.

\section{Material und Methode.}

Es wurden von den Vögeln Taube, Columba livia domestica (ドバト), Eule, Strix ulalensis hondoensis (フクロ) und Huhn, Gallus gallus domesticus (ニワトリ). von den Reptilien Schildkröte, Clemmys japonica (イシガメ) und Schlange, Elaphe quadrivirgata (シマヘビ) und von den Amphibien Kröte, Bufo vulgalis formosus (ヒキガエル), Ochsenfrosch, Rana catesbiana (ウシガエル) und Riesensalamander, Megalobatrachus japonicus (オオサンショウウオ) zur Untersuchung herangezogen. Zur Untersuchung der Struktur der Aorta wurden ferner ein Vogel, Sperlingspapagei, Uroloncha domestica (ジュウシマッ) und eine Amphibie, Rana rugosa (ツチガエル) benutzt. Man bestimmte die Menge der Lipoide im Blut plasma aus dem Herzen nach der einfachen und bebräuchlichen Methode von TOKUDA (1956), nach welcher man das Blutplasma mit dem 20 fachen Volumen 
der physiologischen Kochsalzlösung verdünnte, dies durch ein in $0.05 \%$ iger Viktoriablaulösung gefärbten Filtrierpapier filtrierte und die Farbintensität des Filtrates mit einem Elektrophotometer bestimmte.

Nach der Blutentnahme fixierte man die Tiere für 2 Tage in 10\%iger Formalinlösung. Es wurden Stücke des Knorpels am Wirbelkopf, im Hüftgelenk und zwischen der Sternal- und Vertebralrippe der Vögel und die des Unterteils des Sternums der Amphibien ausgenommen und in Gefrierschnitte von $20 \mu$ Dicke zerlegt. Die miteinander zu vergleichenden Schnitte ließen sich auf ein und demselben Objektglas aufkleben. Man führte die Perjodsäure-SCHIFF-Reaktion (fortan kurz Pjs-SCHIFF-Reaktion) auf Polysaccharide nach dem LILLIEschen Verfahren aus. Auf saure Polysaccharidverbindung prüfte man die metachromatische Färbung in $0.05 \%$ iger Toluidinblaulösung von $\mathrm{pH} 2.5$.

Aus dem mit 10\%igen Formalin fixierten Aortenbogen wurde ein stïck in der Nähe von seinem Ursprung ausgeschnitten. Die $20 \mu$ dicken Gefrierschnitte wurden zur Elastikafärbung mit WEIGERTschem Resorcinfuchsin gefärbt.

\section{Ergebnisse.}

\section{A. Die Menge der Lipoide im Blutplasma.}

Die Resultate sind in der Tabelle zusammengestellt. Sie sind in relativem Wert ausgedrückt, bezogen auf den des normalen Menschen, welcher als 100 angenommen wird. Im allgemeinen ist die Menge der Lipoide im Blutplasma bei den Vögeln und Reptilien größer als bei den Amphibien. Unter den Amphibien ist sie bei der auf dem Lande lebenden Kröte größer als bei dem häufig oder immer im

Die Menge der Lipoide im Blutplasma und die Stärke der Pjs-SCHIFF-Reaktion und der metachromatischen Färbbarkeit mit Toluidinblau der Knorpelgrundsubstanz.

\begin{tabular}{|c|c|c|c|c|c|}
\hline & & $\begin{array}{c}\text { Zahl } \\
\text { d. } \\
\text { Exemplare }\end{array}$ & $\begin{array}{l}\text { Lipoidmenge } \\
\text { im } \\
\text { Blutplasma }\end{array}$ & $\begin{array}{c}\text { Pjs-SCHIFF- } \\
\text { Reaktion d. } \\
\text { Knorpels }\end{array}$ & $\begin{array}{l}\text { Metachro- } \\
\text { masie d. } \\
\text { Knorpels }\end{array}$ \\
\hline \multirow{3}{*}{$\begin{array}{l}\vec{\infty} \\
: \infty \\
: \infty\end{array}$} & $\begin{array}{c}\text { Taube, Columba livia domes } \\
\text { tica }(ト ハ ト)\end{array}$ & 3 & 148 & + & + \\
\hline & $\begin{array}{c}\text { Eule, Strix ulalensis hondo- } \\
\text { ensis (フクロ }\end{array}$ & 2 & 123 & + & + \\
\hline & $\begin{array}{l}\text { Huhn. Gallus gallus domes- } \\
\text { ticus (ニワトリ) }\end{array}$ & 4 & 103 & + & + \\
\hline \multirow{2}{*}{ 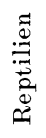 } & $\begin{array}{c}\text { Schlange, Elaphe Quadrivir. } \\
\text { gata (シマへビ) }\end{array}$ & 7 & 126 & + & + \\
\hline & $\begin{array}{c}\text { Schildkröte, Clemmys japo- } \\
\text { nica (イシガ }\end{array}$ & 4 & 120 & ++ & $+t$ \\
\hline \multirow{3}{*}{ 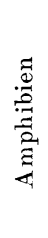 } & $\begin{array}{c}\text { Kröte, Bufo vulgalis formo- } \\
\text { sus }(ヒ キ カ ゙ エ ル)\end{array}$ & 5 & 106 & $t+$ & ++ \\
\hline & $\begin{array}{c}\text { Ochsenfrosch, Rana catesbi- } \\
\text { ana (ウンガエル) }\end{array}$ & 7 & 88 & ++ & ++ \\
\hline & $\begin{array}{c}\text { Riesensalamander, Megalo- } \\
\text { batrachus japonicus } \\
(\text { オホサンン ウウウ }\end{array}$ & 1 & 89 & +++ & ++ \\
\hline
\end{tabular}


Wasser lebenden Ochsenfrosch und Riesensalamander. Die hier angegebenen Mengenverhältnisse der Lipoide stimmen in den großen Zügen mit den von WATANABE (1941) an Vögeln, Reptilien und Amphibien chemisch bestimmten überein, nur daß nach ihm die Lipoidemenge im Blutplasma der schildkröte etwa Affach größser war als die der schlange.

\section{B. Die Pjs-SCHIFF-Reaktion des Knorpels.}

a) Der Knorpel des Hüftgelenkes.

1. Schildköte (カメ) und Riesensalamander (オオサンショウウオ) (Abb. 1).

Die Abb. 1 gibt den Ausfall der Pjs-SCHIFF-Reaktion der senkrechten Schnitte des Hyalinknorpels aus dem Kopf des Femur der Schildkröte und des Riesensalamanders wieder. Die Grundsubstanz des Knorpels färbt sich beim Riesensalamander bei weitem stärker rotviolett als bei der Schildkröte.

2. Kröte (ヒキガエル) und Riesensalamander (オオサンショウウオ) (Abb. 2).

Die Pjs-SCHIFF-Reaktion der Grundsubstanz des Knorpels des Femurkopfes fällt auch beim Riesensalamander viel stärker aus als bei der Kröte.

3. Eule (フクロ) und Ochsenfrosch (ウシガエル).

Der Hüftgelenkknorpel der Eule gehört zu dem Faserknorpel, und der des Ochsenfrosches zu dem Hyalinknorpel. Die Pjs-SCHIFF-Reaktion der Knorpelgrundsubstanz des Femurkopfes ist beim Ochsenfrosch viel stärker als bei der Eule.

4. Huhn (ニワトリ) und Schildkröte (カメ).

Auch der Knorpel des Femurkopfes des Huhnes gehört zu dem Faserknorpel. Seine Pjs-SCHIFF-Reaktion fällt, wie bei der Eule, viel schwächer aus als bei der Schildkröte.

b) Der Knorpel des Wirbelkopfes.

Schlange (ヘビ) und Ochsenfrosch (ウシガエル) (Abb. 3).

Bei den beiden Tieren ist das vordere Ende eines Wirbelkörpers kugelig gestaltet und bildet mit der Pfanne des hinteren Endes des vorder stehenden ein vollkommenes Gelenk. Bei der Schlange ist der Wirbelkopf mit einem ganz dïnnen knorpeligen Lager bedeckt. Die Pjs-SCHIFF-Reaktion der Grundsubstanz des Knorpels tritt bei der Schlange weitaus schwächer ein als beim Ochsenfrosch.

c) Der Rippenknorpel der Vögel und der Sternalknorpel der Amphibien.

1. Huhn (ニワトリ) und Kröte (ヒキガエル) (Abb. 4:).

Beim Huhn gibt es Hyalinknorpel zwischen der Sternal- und Vertebralrippe. Bei der Kröte ist der Schwertfortsatz des Stærnums von Knorpel. Die Pjs-SCHIFFReaktion der Knorpelgrundsubstanz ist bei der Kröte etwas stärker als beim Huhn.

2. Taube (ドバト) und Ochsenfrosch (ウシガエル) (Abb. 5).

Die Pjs-SCHIFF-Reaktion des Knorpels des Schwertfortsatzes des Ochsenfrosches ist in weitem Maße stärker als die des Knorpels zwischen der Sternal- und Vertebralrippe der Taube.

\section{Die metachromatische Färbbarkeit mit Toluidinblau der Knorpelgrundsubstanz.}

Die Grundsubstanz des Hyalin- und Faserknorpels der Vögel, Reptilien und 
Amphibien färbt sich mit $0.05 \%$ iger Toluidinblaulösung von $\mathrm{pH} \mathbf{2 . 5}$ blau mit rotem Ton. Die Stärke der Metachromasie kann aber bei verschiedenen Tieren sehr verschieden sein. Beim Knorpel mit stärkerer Pjs-SCHIFF-Reaktion tritt die Metachromasie stärker ein, wie in der Tabelle zu sehen ist. Sie ist gewöhnlich bei den Vögeln viel schwächer als bei den Amphibien und Reptilien. Besonderes schwach ist die Metachromasie beim Faserknorpel des Hüftgelenkes der Vögel. Dagegen ist sie bei den Amphibien und der Schildkröte in gleichem Ausmaße stark. Bei der Schlange tritt sie etwas schwächer auf.

\section{Die elastischen Fasern in der Wand des Aortenbogens.}

a) Die Vögel.

1. Taube (ハト) (von $0.6 \mathrm{~kg}$ Körpergewicht).

In der quergeschnittenen Media des Aortenbogens der Taube von 9 Monaten erscheinen dicke elastische Lamellen wellenförmig (Abh. 6). Zwischen den Jamellen gibt es fast keine elastischen Fäserchen.

2. Eule (フクロ) (von $1.7 \mathrm{~kg})$.

Wie in Abb. 7 gezeigt ist, sind die elastischen Lamellen in der Media des Aortenbogens dünner als bei der Taube. Zwischen ihnen sieht man feine Fäserchen.

3. Huhn (ニワトリ) (von $1.8 \mathrm{~kg})$.

Die elastischen Elemente im Aortenbogen des Huhns verhalten sich wie bei der Taube und Eule, nur daß die Zwischenräume zwischen den elastischen Lamellen der Media etwas weiter sind, in welchen sich elastische Fäserchen etwas reichlicher finden. Nach IWAKUMA (1957) vermehren sich diese Fäserchen mit dem Lebensalter.

4.. Sperlingspapagei (ジュウシマッ) (von $22 \mathrm{~g}$ ).

Die Aorta dieses Vögelchens von 6 Monaten ist zwar dünnwandig, dementsprechend sind die elastischen Lamellen in der Media sehr dünn. Zu bemerken ist aber, daß swischen den Lamellen sich ganz geringe elastische Fäserchen finden, wie bei den obigen größeren Vögeln.

b) Die Reptilien.

1. Schildkröte (カメ) (von $0.9 \mathrm{~kg}$ ).

In der Media der Aorta befinden sich hauptsächlich longitudinal, zum Teil aber zirkulär laufende Muskelfasern und von ihnen begleitet elastische Fäserchen, so daß die Aortenwand verhältnismäßig dick ist. Die elastischen Lamellen der Media ordnen sich im Gegensatz zu denjenigen der Vögel etwas unregelmäßig und zeigen mehrere Verzweigungen (Abb. 8).

2. Schlange (ヘビ) (von $1.0 \mathrm{~kg}$ ).

Die Wand des Aortenbogens ist sehr dünn. Die elastischen Lamellen in der Media verzweigen sich, wenn auch wenig (Abb. 9).

c) Die Amphibien.

1. Kröte (ヒキガエル) (von $0.29 \mathrm{~kg}$ ).

In der Media sind zahlreiche elastische Fasern vorhanden und fertigen zierliche Netze an, ohne daß sie dicke Lamellen bilden. Vgl. Abb. 10.

2. Ochsenfrosch (ウシガエル) (von $0.31 \mathrm{~kg}$ ). 
Wie in Abb. 10 dargestellt ist, bilden die elastischen Fasern in der Media zierliche Netze.

3. Rana rugosa (ツチガエル) (von $35 \mathrm{~g}$ ).

Die Wand des Aortenbogens dieses Fröschchens ist sehr dünn. In ihr sieht man Netze aus dünnen elastischen Fäserchen.

4.. Riesensalamander (オオサンショウウオ) (von $3.2 \mathrm{~kg}$ ).

Der Schnitt des Aortenbogens des Riesensalamanders ist in Abb. 11 gezeigt.

Die Architektur aus den elastischen Elementen in der Media sieht wie bei den obigen Anuren aus. Man sieht feine elastische Fäserchen in der Media, aber keine wohl gebildeten elastischen Lamellen.

\section{Auswertung.}

Die Lipoidmenge im Blutplasma ist unter den drei Vögeln am größten bei der getreidefressenden Taube, geringer beim Raubvogel Fule und am kleinsten beim omnivoren Huhn. Es besteht also kein Zusammenhang zwischen der Lipoidmenge und Nahrungsart. Diese Tatsache stimmt nicht mit dem Befunde von WATANABE (191:0) überein, nach welchem bei Säugetieren die Lipoidmenge im Blutplasma von Karnivoren höher ist als von Herbivoren und die von Omnivoren dazwischen steht.

Bei den Amphibien ist die Menge der Lipoide im Blutplasma geringer als bei den Reptilien, und besonders gering bei dem im Wasser lebenden Ochsenfrosch und Riesensalamander. Dies steht vielleicht damit im Zusammenhang, daß die Haut der Amphibien für Wasser wohl durchläßig ist, und, daß der Wassergehalt des Blutes bei den Amphibien höher ist als bei den Vögeln und Reptilien und unter den Amphibien beim Frosch höher ist als bei der Kröte, was von SCHULZ und KR̈̈GER (1925) konstatiert wurde.

Die Pjs-SCHIFF-Reaktion und die metachromatische Färbung mit Toluidinblau der Knorpelgrundsubstanz erfolgen, wie aus der Tabelle ersehen ist, bei den Amphibien und dem Reptil Schildkröte stärker als bei den Vögeln und dem Reptil Schlange. Die Knorpelgrundsubstanz ist also bei den ersteren an sauren Polysaccharidverbindungen reich. Was die Beziehung zwischen der Menge von sauren Polysaccharidverbindungen in der Knorpelgrundsubstanz und der von Lipoiden im Blutplasma betrifft, so sind die ersteren geringer bei den an Blutplasmalipoiden reichen Tieren, Taube, Eule und Schlange, dagegen sind sie reicher bei den an Plasmalipoiden armen Tieren, Kröte, Ochsenfrosch und Riesensalamander. Auf einer solchen Tatsache hat schon TOKUDA (1957) an den Knorpel- und Knochenfischen aufmerksam gemacht. Nach ihm sind die Lipoide im Blutplasma bei Knorpelfischen beträchtlich geringer vorhanden als bei Knochenfischen, dagegen finden sich in der Knorpelgrundsubstanz der ersteren die sauren Polysaccharidverbindungen in weitaus größerer Menge als in der der letzteren.

In der Media der Aortenwand der Vögel sind schöne elastische Lamellen gebildet. Bei den Reptilien und Amphibien und ferner, wie früher von dem Verfasser (1957) bemerkt ist, bei den niedrigsten Wirbeltieren Fischen verzweigen sie sich immer mehr und bilden ein elastisches Netzwerk. Die Körpergröße der Tiere ist dabei fast belanglos. WALDYER (1927) hat auch schon bei den Reptilien und 
Amphibien ungefähr Gleiches beobachtet.

\section{Zusammenfassung.}

1. Nach der Untersuchung an drei Vögeln, zwei Reptilien und drei Amphibien ist die Lipoidmenge im Blutplasma in der Regel am größten bei den Vögeln, die Menge von sauren Polysaccharidverbindungen in der Knorpelgrundsubstanz aber hei ihnen am geringsten. Ferner, die Lipoide im Blutplasma lassen sich bei den Reptilien mehr nachweisen als bei den Amphihien, die sauren Polysaccharidverbindungen im Knorpel aber umgekehrt.

2. Die Architektur der elastischen Elemente in der Aortenwand wurde bei vier Vögeln, zwei Reptilien und vier Amphibien untersucht. Bei den Vögeln bilden sich regelmäßig angeordnete, wenig verzweigte dicke elastische Lamellen in der Media, dagegen sind die elastischen Elemente bei der niedrigeren Tieren, außer bei der Schlange, immer stärker verzweigt und bilden ein dichteres Netzwerk.

\section{内 容 自 抄。}

向漿類脂質の量が鳥類と爬虫類に多く, 両棲類に少いのに反し, 軟骨基質の過 ヨード酸-Schiff 反忘とトルイヂン青での異調染色性にて証明される酸性多糖類 化合物は前 2 者に少なく，後者に多い.

鳥類では大動脈弓の中膜に分枝の少ない厚い弾性板ができており，爬虫類では 弾性板に細い分枝が多く，更に雨棲類と下等になるに企って分枝がますます多く なり，密な弾性網が作られている。

\section{Literatur.}

Dauber, D.V. and L. N.Katz: Experimental cholesterol atheromatosis in an omunivorous animal, the chick. Arch. Path. (Am.) 34 (1942). - Hahn, F.: Abolishment of alimentary lipemia following injection of heparin. Science 98 (1943). - Iwakuma, H. : Elastische Lamellen in der Media der Brustaorta des Huhnes während der prä- und postnatalen Entwicklung. Arch. hist. jap. 13 (1957). - Kitade, T. : Über die Altersveränderungen der Aorta ventralis von Teleostir Goldfish und über die Verschiedenheiten der Struktur der Aorta dorsalis von Selachier und Teleotier. Arch. hist. jap. 13 (1957). - Kurita, H.: Über den Einfluß von Chondroitinsulfat auf die experimentelle Atherosklerose beim Kaninchen. (Jap.) Med. J. Shinshu Univ. 1 (1955). - Miyao, S. u. a.: Der Einfluß von Chondroitinsulfat auf die experimentelle Atherosklerose beim Haushuhn. (Jap.) Naika no Riyoiki 5 (1957). - Schulz, Fr. N. u. F. V. Krüger: Das Blut der Wirbeltiere. Wintersteins Handbuch der vergleichenden Physiologie. Bd. 1. 1925. - Tokuda, M. : Eine neue Methode zur Bestimmung des Lipoides im Blutplasma durch Filtrierung mit Viktoriablau getränktem Filtrierpapier. Arch. hist. jap. 10 (1956). Über die Menge der Lipoide im Blut. plasma von Knochen- und Knorpelfisch und die Stärke der Pjs-Schiff-Reaktion des Knorpels und seiner Metachromasie mit Toluidinblau. Arch. hist. jap. 12 (1957). — Waldyer, A. : Der Bau der Aortenwand bei Amphihien und Reptilien. Z. mikr.-anat. Forsch. 11 (1927). -

Watanabe, R. : The distribution of lipoids in the blood serum and red blood cells of various kinds of animals. Keijo J. Med. 10 (1940); 11 (1941). 
Tafelabbildungen. 


\section{Erläuterung $z u$ den Tafelabbildung.}

Abb. 1. Knorpel aus dem Hüftgelenk von Schildkröte, Clemmys japonica (インガメ) und Riesensalamander, Megalobatrachus japonicus (オホサンショウウオ). Pjs-SCHIFFReaktion. $100 \times$

Abb. 2. Knorpel aus dem Hüftgelenk von Kröte, Bufo vulgaris formosus (ヒキカエ ル) und von Riesensalamander, Megalobatrachus japonicus (オホサンショウウオ). PjsSCHIFF-Reaktion. $100 \times$

Abb. 3. Knorpel aus dem Caput vertebrae von Schlange, Elaphe quadrivirgata (シマ へビ) und Ochsenfrosch, Rana catesbiana (ウンガエル). Pjs-SCHIFF-Reaktion. $100 \times$ 
T. KIT:ADA (1)
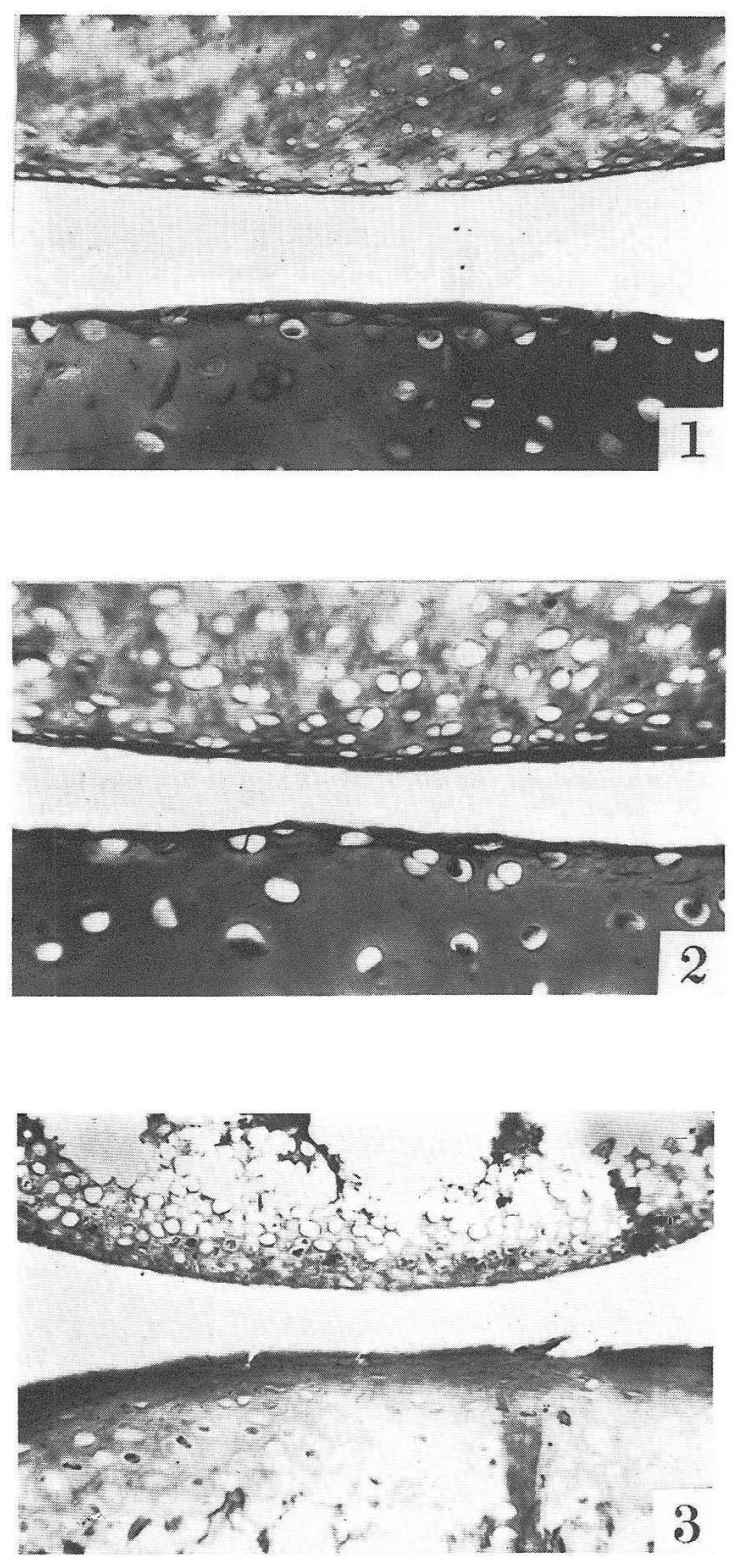
Abb. 4. Rippenknorpel von Huhn, Gallus gallus domesticus (ニワトリ) und Knorpel aus dem Sternum von Kröte. Bufo vulgaris formosus (ヒキガエル). Pjs-SCHIFFReaktion. $100 \times$

Abb. 5. Rippenknorpel von Taube, Columba livia domestica (ドハト) und Knorpel aus dem Sternum von Ochsenf rosch, Rana catesbiana (ウンカエル). Pjs-SCHIFF-Reaktion. $100 \times$ 
T. KITAD ( 2$)$
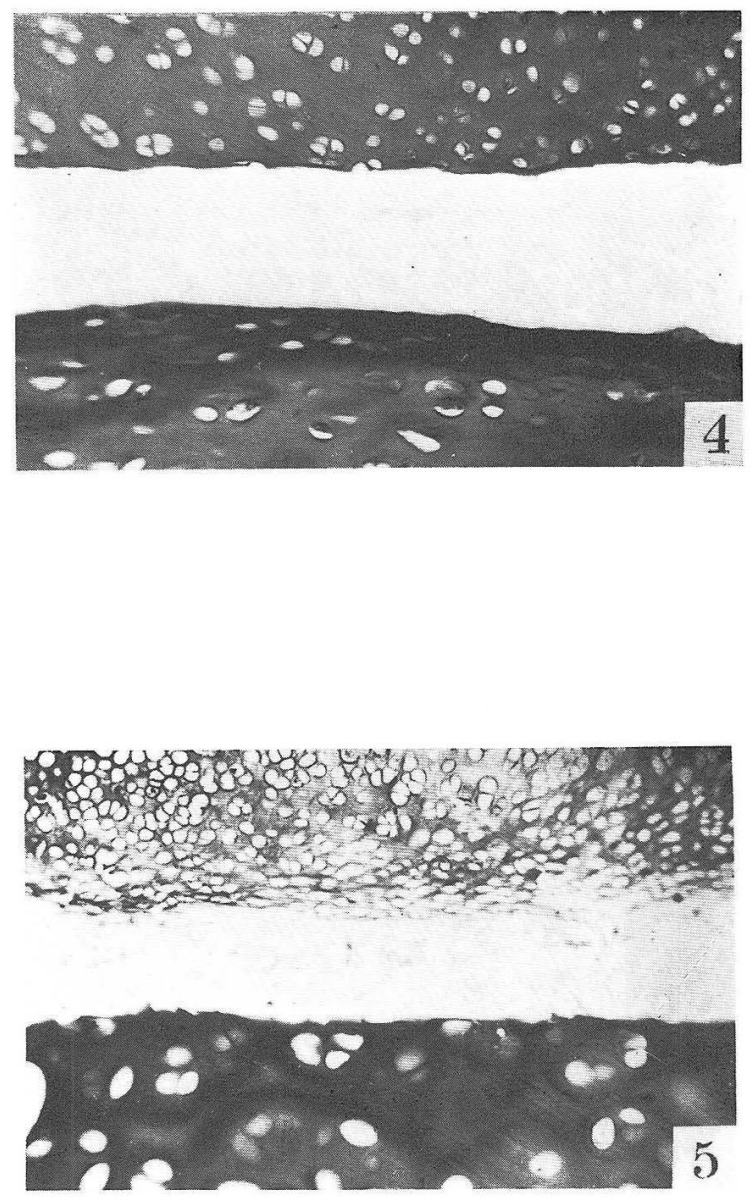
Abb. 6-11. Querschnitt des Aortenbogens. Färbung mit Resorcinfuchsin. $200 \times$ Abb. 6. Von Taube, Columba livia domestica (ドハト).

Abb. 7. Von Eule, Strix uralensis hondoensis (フクロ).

Abb. 8. Von Schildkröte. Clemmys japonica (インガメ).

Abb. 9. Von Schlange, Elaphe quadrivirgata (ンマーピ).

Abb. 10. Von Ochsenfrosch, Rana catesbiana (ウンガエル).

Abb. 11. Von Riesensalamander, Megalobatrachus japonicus (オホサンショウウオ). 
T. KITADA (3)

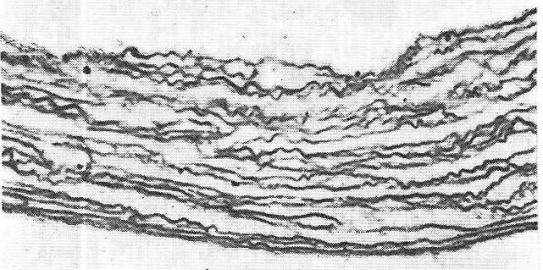

6

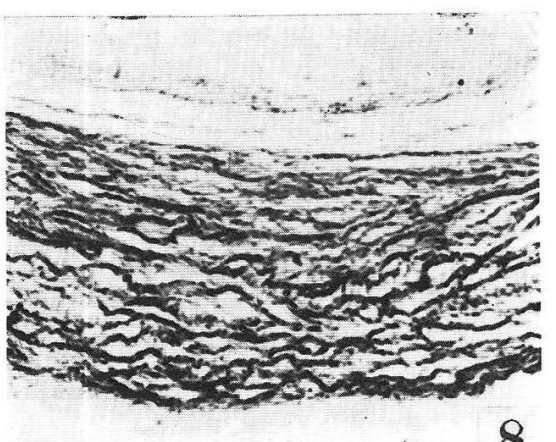

8

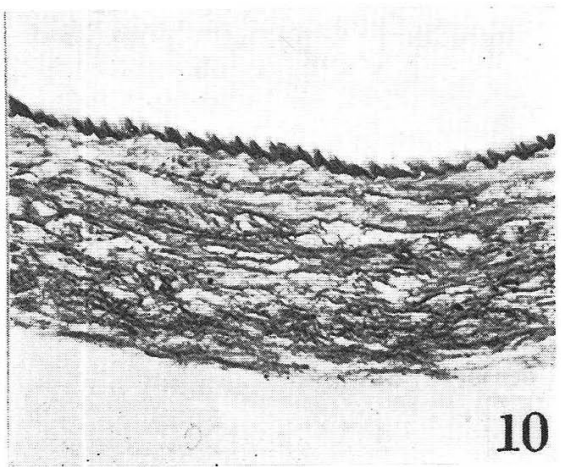

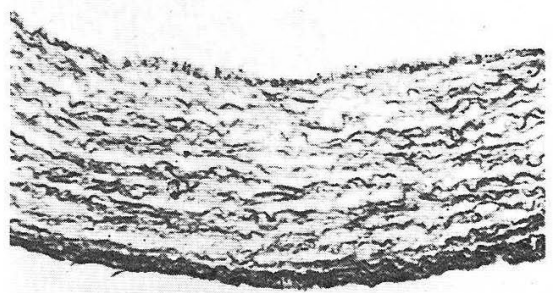

7 